\title{
LA SATISFACCIÓN DOCENTE Y SU INFLUENCIA EN EL DESEMPEÑO LABORAL EN EL CENTRO DE IDIOMAS DE LA UNIVERSIDAD NACIONAL JORGE BASADRE GROHMANN DE TACNA, EN EL AÑO 2014
}

\section{Teacher Satisfaction and its Influence on the Job Performance in the Center of Languages of Jorge Basadre Grohmann National University of Tacna, 2014}

\section{Rey Ángel Linares Dávalos 5}

\section{RESUMEN}

Objetivo: El trabajo de investigación tuvo como objetivo principal el determinar la influencia de la satisfacción docente sobre el desempeño laboral en el Centro de Idiomas de la Universidad Nacional Jorge Basadre Grohmann de Tacna de Tacna, en el año 2014.

Método: El trabajo de campo abarcó a la población de docentes, es decir 45 personas. El tipo de investigación fue aplicada, de características descriptivas y correlacionales (puesto que se utilizaron modelos ya existentes que permitieron efectuar una análisis descriptivo del comportamiento de las variables, para luego relacionarlos); el diseño de la investigación fue no experimental, cuya recolección de información fue de corte transversal (dado que se efectuaron algunas recomendaciones y los datos se recabaron en un momento del tiempo).

Resultados: Se encontró que no existe una influencia significativa de la satisfacción docente sobre el desempeño laboral. Además, el personal docente considera que se caracteriza por un nivel aceptable de

\footnotetext{
${ }^{5}$ Magister en Docencia Universitaria y Gestión Educativa
}

satisfacción; el indicador más destacado fue la "Supervisión", siendo los aspectos a mejorar las "Recompensas justas" y las "Condiciones de trabajo".

Conclusiones: El personal docente considera que su nivel de desempeño en sus labores académicas son destacadas, el indicador más resaltado fue la "Satisfacción al estudiante", siendo los aspectos a mejorar la "Labor de equipo" y el "Crecimiento personal".

\section{PALABRAS CLAVES}

Dirección, Eficiencia, Eficacia, Ética, Evaluación de desempeño, Habilidad, Motivación, Liderazgo

\section{ABSTRACT}

Objective: The research's main objective was to determine the influence of teacher satisfaction with job performance in the Language Centre of the National University Jorge Basadre Grohmann Tacna Tacna in 2014.

Method: The field work included teaching the population, ie 45 people. The research was applied, descriptive and correlational features (since they allow existing models to make a descriptive analysis of the behavior of the variables, and then relate them used); The research design was not experimental, whose collection of information was crosssectional (given that some recommendations were made and 
the data were collected at a point in time).

Results: We found that there is no significant influence of teacher satisfaction with job performance. In addition, staff believes that is characterized by an acceptable level of satisfaction; the most prominent indicator was the "Monitoring", with areas for improvement "just rewards" and "working conditions".
Conclusions: The staff believes that its level of performance in their academic work are outstanding, the indicator highlighted was the "student satisfaction", being the aspects to improve the "teamwork" and "personal growth".

\section{KEYWORDS}

Management, effectiveness, evaluation, Leadership efficiency, ethics, performance Skill, Motivation,

\section{INTRODUCCIÓN}

La satisfacción docente es uno de los aspectos prioritarios que una institución educativa debe buscar. Buscar que los docentes manifiesten su satisfacción con el rigor de los cursos, con las interacciones con los colegas y estudiantes, con el apoyo a la dirección en el fortalecimiento de sus capacidades, con la colaboración a las instalaciones y equipamiento que apoyan dichas interacciones. El problema se encuentra al momento de ofrecer los servicios educativos a los estudiantes, como una educación de calidad, es difícil decidir entre llenar o satisfacer las expectativas del estudiante o las del mercado que requerirá de sus servicios o la sociedad que demandara sus capacidades cuando se conviertan en egresados.

La pertenencia a un grupo, el apoyo recibido por los compañeros, o la oportunidad de trabajar en equipo, son factores que pueden proporcionar elevada gratificación y producir satisfacción y bienestar, constituyendo elementos motivacionales que previenen o reducen la insatisfacción laboral.

Cuando se produce un contexto de insatisfacción en una Entidad Educativa, éste afecta aspectos muy importantes como productividad, rendimiento, entre otros. En una Institución como el Centro de Idiomas de la UNJBG - Tacna, actualmente enmarcada en un mercado competitivo relacionado con el proporcionar servicios de formación en idiomas, resulta necesario y ventajoso, captar a los estudiantes por la imagen que proyecta la institución como entidad educativa formadora de personas que se insertan en el mercado laboral o que amplían sus horizontes en el ámbito académico.

En base a la experiencia laboral, y sobre todo a la comunicación con algunos trabajadores, directivos y profesores de dicha entidad, percibo que ciclo a ciclo existe una marcada rotación de los estudiantes, y ello generado principalmente porque su percepción para decidir el continuar en dicha entidad se basa en la calidad de la plana docente, el equipamiento tecnológico e instalaciones y ambiente general de la institución, dichos aspectos no están implementados de forma óptima en el CEID - UNJBG; se agrega a ello, que el profesor no cuenta con la estabilidad laboral que le permita mejorar sus niveles de compromiso con la entidad, pues en cada ciclo puede sufrir una modificación de su carga horaria. 
Algunos autores definieron satisfacción por el trabajo como "El resultado de diversas actitudes que tienen los trabajadores en relación al salario, la supervisión, el reconocimiento, oportunidades de ascenso (entre otros) ligados a otros factores como la edad, la salud, relaciones familiares, posición social, recreación y demás actividades en organizaciones laborales, políticas y sociales"(BLUM \& NAYLOR, 1999).

También que la "Satisfacción en el trabajo es la consecuencia de varias actitudes que el trabajador guarda hacia su trabajo, hacia factores afines y hacia la vida en general".(VON HALLER, 1999).

Para Robbins es "La actitud del trabajador frente a su propio trabajo, dicha actitud está basada en las creencias y valores que el trabajador desarrolla de su propio trabajo". Las actitudes son determinadas conjuntamente por las características actuales del puesto como por las percepciones que tiene el trabajador de lo que "deberían ser". Generalmente las tres clases de características del empleado que afectan las percepciones del "debería ser" (lo que desea un empleado de su puesto) son: Las necesidades, los valores y rasgos personales.(ROBBINS S. , 2004).

Una postura concibe que la satisfacción de los trabajadores es producto de una actitud positiva hacia factores no necesariamente ligados estrechamente a la tarea que desempeña, comúnmente llamados factores extrínsecos o que giran en torno a la actividad laboral. Ejemplo de estos factores son el salario, las condiciones de trabajo, la estabilidad laboral, las relaciones con los compañeros de trabajo, supervisores y subordinados, entre otros (RAMIREZ, 2006).

La satisfacción laboral no es más que el estado de ánimo que se genera con respecto a los factores que compensan la actividad realizada, expresada en salarios, condiciones de trabajo y estatus (MORSE, 1953). En ese mismo sentido, se plantea que es la seguridad en el empleo (estabilidad), el ambiente de trabajo y la competencia demostrada por los supervisores, lo que podría generar sentimientos de agrado o desagrado con respecto al trabajo (PERRY, 1961). Asimismo, otros autores sostienen que para lograr un alto rendimiento en el trabajo, los trabajadores deben estar altamente motivados y esto se logra a través de incentivos externos tales como una buena remuneración, excelentes condiciones de trabajo y pagos compensatorios paralelos al salario (PORTER \& LAWLER, 1999), (WANOUS, 1975).

Maslow, máximo exponente de esta corriente, sostiene que el hombre se encontrará más satisfecho mientras más se acerque a la fase de autorrealización, siendo el trabajo la actividad que más posibilidades brinda al hombre para ingresar a esa fase por los retos asociados a éste (interesante, creativo y significativo) y que motivan al logro(RAMIREZ, 2006). En esta línea de pensamiento, otros autores: sostienen que el trabajador mientras perciba que su trabajo sea interesante y significativo, se sienta responsable por la calidad de sus productos (bienes o servicios), se sienta identificado por el resultado de su esfuerzo y asuma que se respeta y valora sus prescripciones, se sentirá motivado por los logros alcanzados (HACKMAN \& LAWLER, 1971), (MCGREGOR, 1972), (RAMIREZ, 2006).

Los trabajadores que están orientados hacia el logro, son más eficaces y tienen alto rendimiento en sus metas individuales si perciben que su trabajo les ofrece 
responsabilidad personal, retroalimentación y riesgos moderados. Lo que no sucede si el estímulo está limitado a las compensaciones económicas. (ROBBINS S. , 1999).

Otra posición, que da un vuelco a las interpretaciones anteriores sobre los factores generadores de satisfacción laboral, es la sostenida por la Teoría Bifactorial de Herzberg y colaboradores (1959). (HERZBERG, MAUSNER, \& SNYDERMAN, 1959). Fundamentan parte de sus apreciaciones la Teoría de Maslow sobre las necesidades higiénicas y de autorrealización del hombre. Así, el hombre tiene un conjunto de necesidades biológicamente básicas (hambre, sexo, etc.), y un conjunto de necesidades referentes a una característica particular del ser humano como es la capacidad de logro y, a través de ésta, el crecimiento psicológico. Ambos son dos sistemas distintos de necesidades humanas. Las necesidades básicas de supervivencia son satisfechas instintiva o conscientemente a través de conductas aprendidas natural o socialmente (la cacería, la pesca, el trabajo como vía para obtener dinero y poder adquirir alimentos). El otro conjunto de necesidades está ligado a la necesidad de crecimiento personal y de autorrealización. La satisfacción del primer conjunto de necesidades o factores higiénicos, según la Teoría Bifactorial, previene la insatisfacción pero no genera satisfacción positiva; en cambio los factores motivadores sí generan satisfacción. (RAMIREZ, 2006).

Así, según esta teoría, en el mundo laboral existirá satisfacción e insatisfacción, en función de que las necesidades del individuo estén o no cubiertas en la situación laboral. En estos casos los factores de satisfacción o "Motivadores" se diferencian claramente: son intrínsecos al trabajo y pueden concretarse en el gusto por el trabajo mismo, la responsabilidad que deriva del mismo, el deseo de realización o de logro, el de obtener la estima ajena y la propia promoción. Estos factores son considerados como de verdadera satisfacción positiva. Una segunda agrupación se hace entre algunos factores que "Rodean" al trabajo (extrínsecos), tales como: condiciones materiales de seguridad e higiene, salario, política de personal, condiciones de trabajo, administración de la organización y relación entre colegas.

Esperanza Pedraza señala que el desempeño laboral, se define como "Aquellas acciones o comportamientos observados en los empleados que son relevantes para los objetivos de la organización, y que pueden ser medidos en términos de las competencias de cada individuo y su nivel de contribución a la empresa" (PEDRAZA, AMAYA, \& CONDE, 2010).

Por esta razón, la evaluación del desempeño, es un proceso destinado a determinar y comunicar a los empleados, la forma en que están desempeñando su trabajo y a elaborar planes de mejora .Cuando se realiza adecuadamente la evaluación de personal no solo hacen saber a los empleados cuál es su nivel de cumplimiento, sino que influyen en su nivel futuro de esfuerzo y en el desempeño correcto de sus tareas.

Los componentes para un buen desempeño laboral son: Un buen ambiente externo; la motivación de la organización: y la capacidad de la organización.

La evaluación del desempeño no es un fin en sí mismo, sino un instrumento, una herramienta para mejorar los resultados de los recursos humanos de la empresa. Los superiores jerárquicos están siempre observando la forma en que los 
empleados desempeñan sus tareas y se están formando impresiones acerca de su valor relativo para la organización (ACTIO REPORTE, 2006).

La mayoría de las organizaciones grandes han creado un programa formal, diseñado para facilitar y estandarizar la evaluación de los empleados; sin embargo, resulta poco trabajada la evaluación a nivel de pequeña y mediana empresa (ACTIO REPORTE, 2006).

Se considera que la evaluación del desempeño es "Un procedimiento continuo, sistemático, orgánico y en cascada, de expresión de juicios acerca del personal de una empresa, en relación con su trabajo habitual, que pretende sustituir a los juicios ocasionales y formulados de acuerdo con los más variados criterios" (GONZALES, 2009). Según este autor, la evaluación se produce de forma vertical, cada persona es evaluada por su jefe inmediato y éste, a su vez, es evaluado por un jefe de un nivel superior. De esta forma, el máximo responsable (gerente) sería sólo evaluador y en el nivel más bajo, las personas serían evaluadas pero no serían evaluadores.

\section{MATERIALES Y MÉTODOS}

El tipo de investigación es aplicada (se utilizaron enfoques o modelos teóricos ya existentes), siendo el nivel de investigación descriptivo y correlacional. El diseño de la investigación es no experimental, cuya recolección de información fue de corte transversal. La población de docentes es de 45. Puesto que es número manejable, se efectuó un censo; es decir, en el presente año de estudios (2014) se encuestaron a los 45 docentes.

La variable independiente es la satisfacción docente, y la variable dependiente es el desempeño laboral; se procede a presentar la operacionalización de las variables. Los instrumentos fueron confeccionados por el investigador, en base a la revisión bibliográfica y el apoyo del asesor; los mismos fueron validados por el Alpha de Cronbach. Se utilizaron dos instrumentos: Un cuestionario para determinar el nivel de satisfacción que caracteriza al docente. Un cuestionario de autoevaluación para determinar el nivel de desempeño laboral. La fuente de información fue esencialmente primaria, puesto que fue recabada de los docentes del Centro de Idiomas de la UNJBG de Tacna. El marco teórico desarrollado, implicó fuentes bibliográficas, tanto escritas como de medios electrónicos.

Puesto que el objetivo de la tesis fue determinar la relación de una variable sobre otra, se aplicaron ambos instrumentos (el cuestionario para determinar el nivel de satisfacción que caracteriza al docente y el cuestionario de autoevaluación para determinar el nivel de desempeño laboral), a la misma unidad de análisis (el docente del CEID - UNJBG de Tacna)(ALVAREZ BECERRA, 2015). Asimismo, se construyeron ambos instrumentos debido a que no se encontró uno validado y que sea afín al trabajo realizado, con el acompañamiento del asesor y con la verificación de su confiabilidad, resultando esta ser adecuada.

El procesamiento de los datos se hizo en base al Microsoft Excel y al software estadístico SPSS versión 20,0 en español. 


\section{RESULTADOS}

\section{Análisis integral}

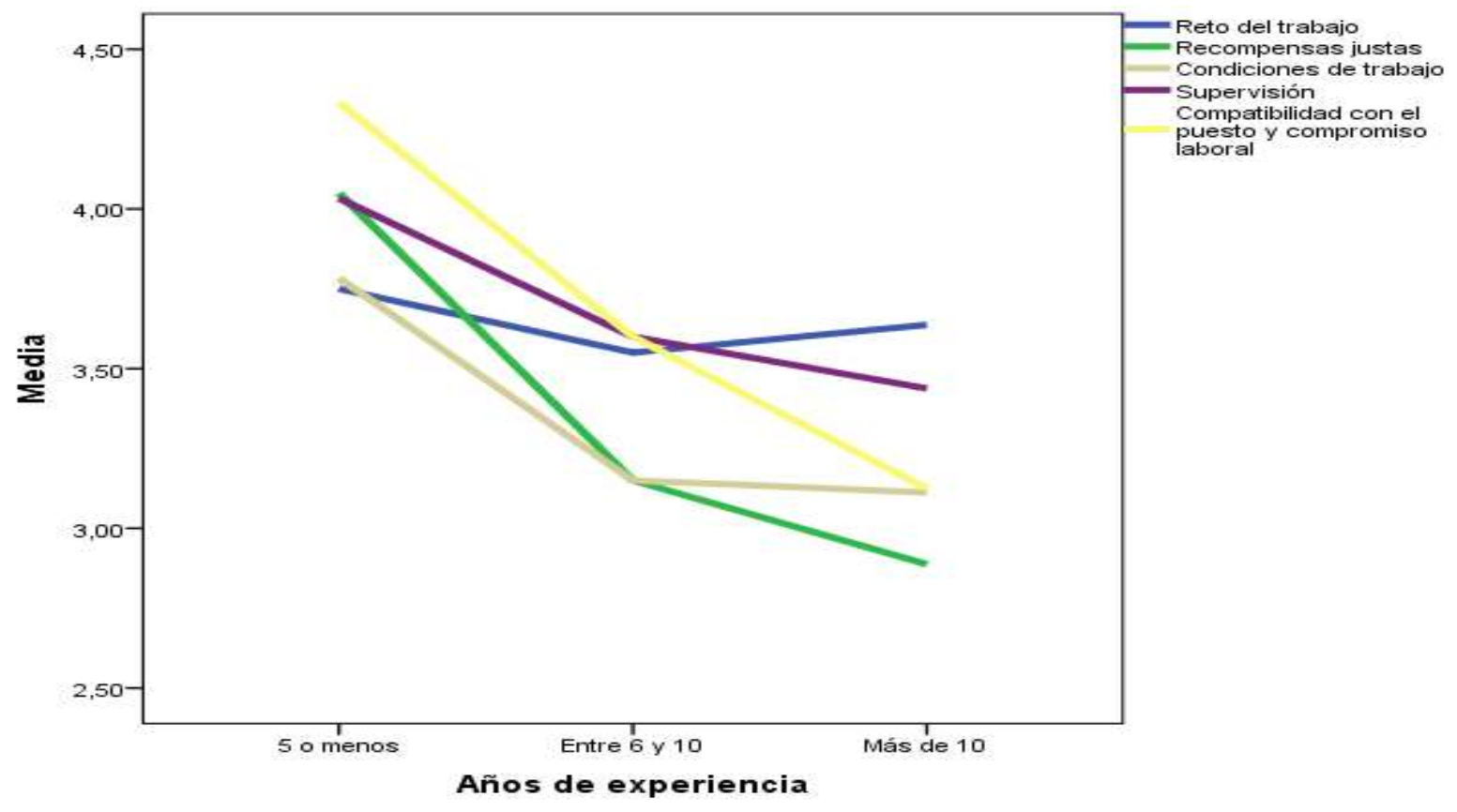

FUENTE: Cuestionario de "Satisfacción Docente"

\section{Figura $\mathbf{N}^{\circ} 1$}

\section{Satisfacción del docente}

La figura $\mathrm{N}^{\circ} 1$ nos permite efectuar un análisis integral, de donde se obtuvo un valor medio de 70,56 y una desviación típica de 13,975, el cual al ubicarlo en la escala de valoración previamente identificada, cae en la intervalo denominado "Alto nivel de satisfacción docente"; lo que permite concluir que el personal docente del CEID - UNJBG de Tacna considera que se caracteriza por un nivel aceptable de satisfacción por la docencia que imparte en dicha entidad. Al analizar por niveles y de forma específica, se encontró que son 18 los que precisan tener un alto nivel de satisfacción, son 12 los que señalan tener un nivel regular, 10 con un alto nivel, y son 5 los que señalan tener un nivel bajo de satisfacción docente. Se observa que al aumentar los años de experiencia docente disminuye la satisfacción docente. Asimismo, al comparar por género, son las docentes mujeres las que destacan más el comportamiento de dicha variable.

Al analizar por cada uno de los indicadores, se encontró que el aspecto más resaltado fue la "Supervisión", siendo los aspectos a mejorar las "Recompensas justas" y las "Condiciones de trabajo". 


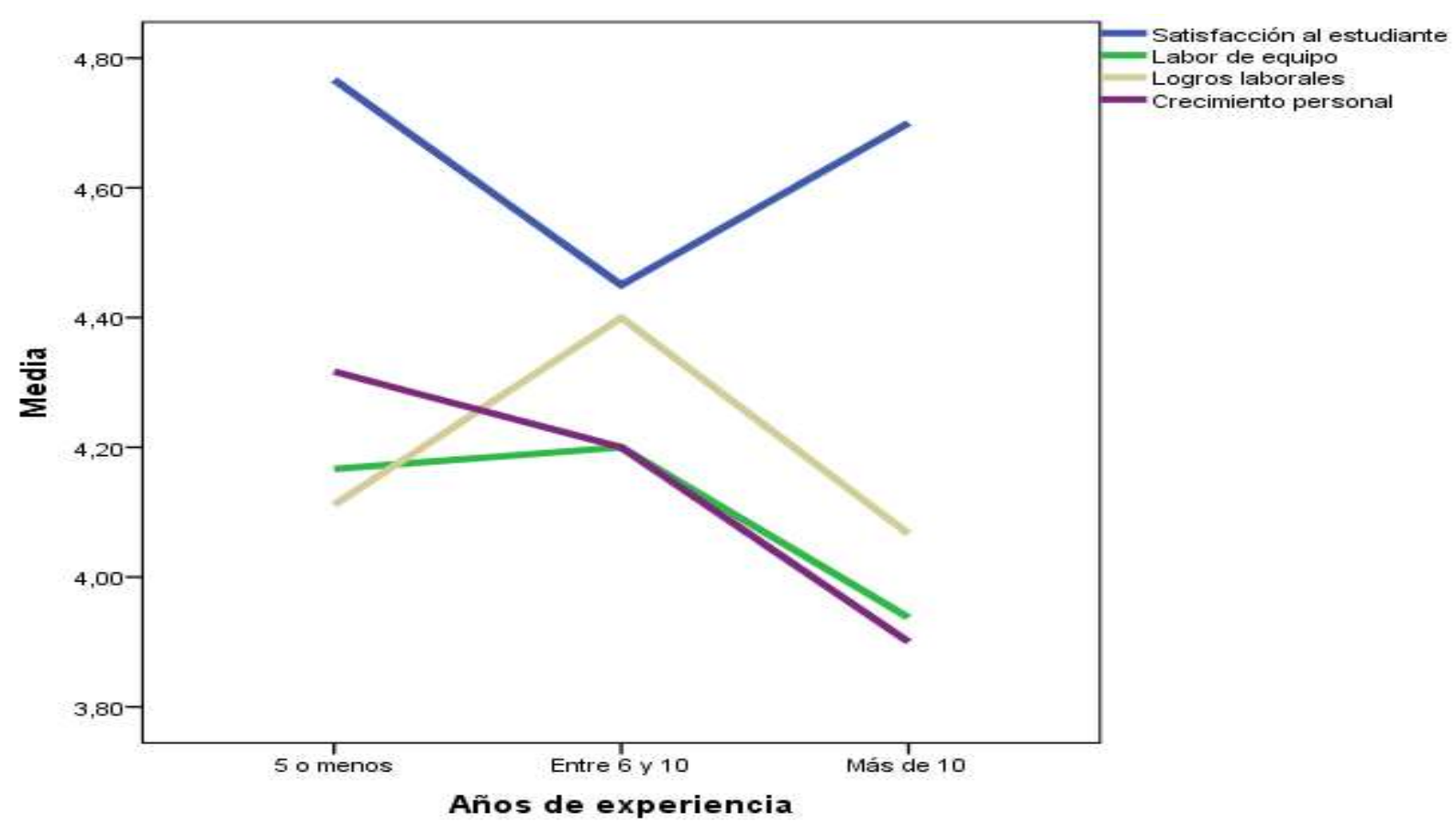

FUENTE: Cuestionario de "Desempeño laboral"

Figura $\mathbf{N}^{\circ} 2$

\section{Desempeño laboral}

La figura $\mathrm{N}^{\circ} 2$ nos permite efectuar un análisis integral, de donde se obtuvo un valor medio de 63,84 y una desviación típica de 4,467, el cual al ubicarlo en la escala de valoración previamente identificada, cae en la intervalo denominado "Nivel de desempeño laboral muy adecuado; lo que permite concluir que el personal docente del CEID - UNJBG de Tacna considera que su nivel de desempeño y desenvolvimiento de sus laborales académicos en la entidad son destacadas. Al analizar por niveles y de forma específica, se encontró que son 26 los que precisan tener un nivel muy adecuado, y son 19 los que señalan presentar un nivel adecuado. Al observar los años de experiencia, los indicadores "crecimiento personal" y "labor de equipo", disminuyen su valoración conforme va aumentando el tiempo; "satisfacción al estudiante" y "logros laborales" tienen un comportamiento irregular y opuesto. Asimismo, al comparar por género, son las docentes mujeres las que destacan más el comportamiento de dicha variable.

Al analizar por cada uno de los indicadores, se encontró que el aspecto más resaltado fue la "Satisfacción al estudiante", siendo los aspectos a mejorar la "Labor de equipo" y el "Crecimiento personal". 


\section{Contraste de las hipótesis sobre las variables}

Sobre la satisfacción del docente analizado, que cuenta con un valor medio general de 70,56 y una desviación típica de 13,975; que permitió concluir que el personal docente del CEID - UNJBG de Tacna considera que se caracteriza por un nivel aceptable de satisfacción por la docencia que imparte en dicha entidad. Para comprobar ello, se aplicó una prueba de hipótesis para la media, para lo cual se procede a plantear las siguientes hipótesis, de donde:

\section{$H_{0}: \mu \geq 52$ (satisfacción docente regular, alta o muy alta) $H_{1}: \mu<52$ (satisfacción docente baja o muy baja)}

Donde $\mu$ representa el valor medio del nivel de satisfacción docente; se reemplaza en la fórmula:

$$
t=\frac{\bar{x}-\mu_{\bar{x}}}{\frac{s}{\sqrt{n}}}
$$

$$
\mathrm{t}=(70,56-52) /\left(13,975 / 45^{\wedge 1} / 2\right)=5,07
$$

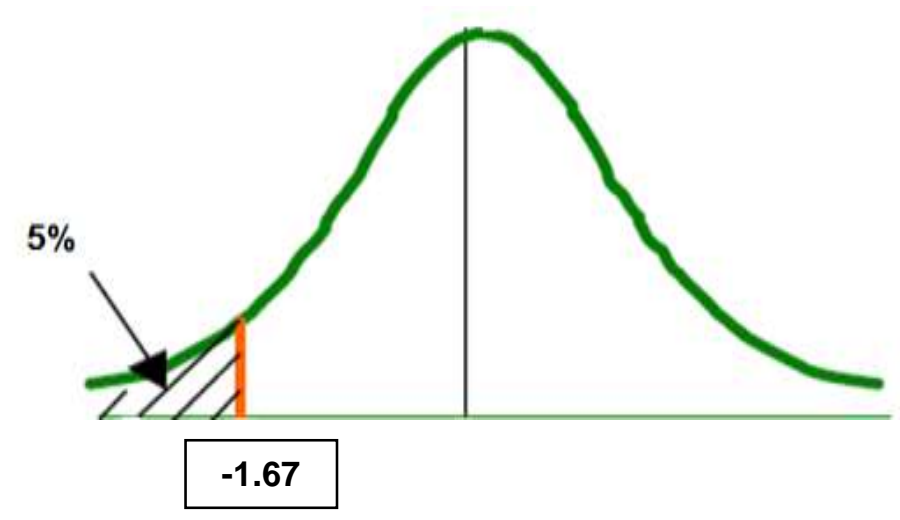

El valor de $t$ de tabla al $5 \%$ de significancia es de $-1,67$, el $t$ calculado $=$

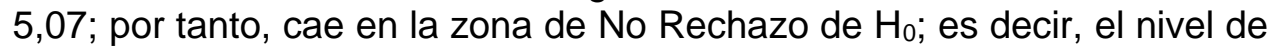
satisfacción docente que caracteriza al Centro de Idiomas de la UNJBG de Tacna no es bajo.

Sobre el desempeño laboral analizada, que cuenta con un valor medio general de 63,84 y una desviación típica de 4,467; que permitió concluir que el personal docente del CEID - UNJBG de Tacna considera que su nivel de desempeño y desenvolvimiento de sus laborales académicos en la entidad son destacadas. A través de la prueba de hipótesis para la media, se procede a contrastar dicha hipótesis, de donde: 


\section{$\mathrm{H}_{0}: \quad \mu \quad \geq 51$ (desempeño laboral adecuada o muy adecuada) \\ $\mathrm{H}_{1}$ : $\quad \mu<51$ (desempeño laboral regular, inadecuada o muy inadecuada)}

Donde $\mu$ representa el valor medio del nivel de desempeño laboral; se reemplaza en la fórmula:

$$
t=\frac{\bar{x}-\mu_{\bar{x}}}{\frac{s}{\sqrt{n}}}
$$$$
t=(63,84-51) /\left(4,467 / 45^{\wedge 1} / 2\right)=19,28
$$

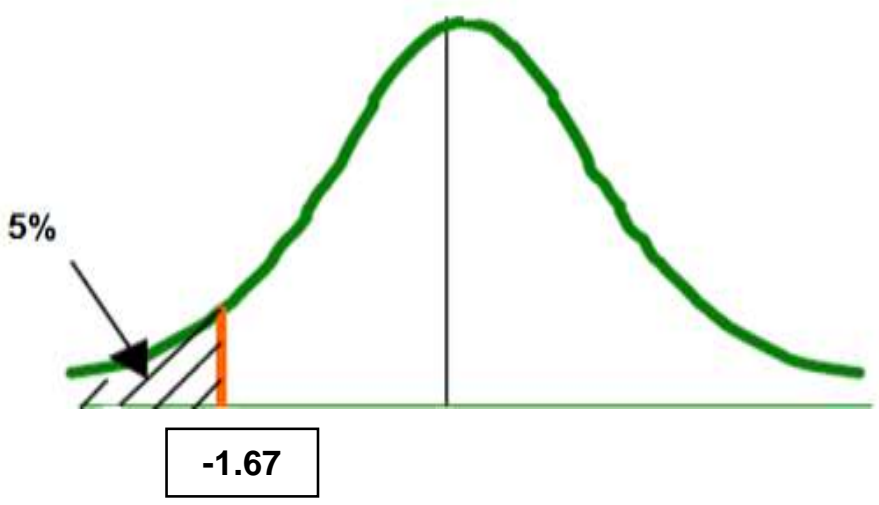

El valor de $t$ de tabla al $5 \%$ de significancia es de $-1,67$, el $t$ calculado $=$ 19,28; por tanto, cae en la zona de No Rechazo de $\mathrm{H}_{0}$; es decir, el desempeño laboral que caracteriza a los docentes del Centro de Idiomas de la UNJBG de Tacna no es regular.

Para el contraste de la hipótesis general, se utilizó el estadístico chicuadrado; cuyos resultados se indican en la siguiente tabla resumen:

$\mathrm{H}_{0}$ : No existe influencia significativa

$\mathrm{H}_{1}$ : Existe influencia significativa

Tabla $\mathrm{N}^{\circ} 1$

ESTADÍSTICO CHI-CUADRADO

\begin{tabular}{|c|c|c|c|c|c|c|}
\hline & \multicolumn{4}{|c|}{ Satisfacción docente } & \multirow[t]{2}{*}{ Total } \\
\hline & & Baja & Regular & Alta & Muy alta & \\
\hline \multirow{2}{*}{\multicolumn{2}{|c|}{$\begin{aligned} \text { Desempeño laboral } & \text { Adecuado } \\
\text { Total } & \text { Muy adecuado }\end{aligned}$}} & 3 & 5 & 6 & 5 & 19 \\
\hline & & $\begin{array}{l}2 \\
5\end{array}$ & $\begin{array}{c}7 \\
12\end{array}$ & $\begin{array}{l}12 \\
18\end{array}$ & $\begin{array}{c}5 \\
10\end{array}$ & $\begin{array}{l}26 \\
45\end{array}$ \\
\hline
\end{tabular}


Pruebas de chi-cuadrado

\begin{tabular}{|l|r|r|r|}
\hline & \multicolumn{1}{|c|}{ Valor } & gl & \multicolumn{1}{|c|}{$\begin{array}{c}\text { Sig. asintótica } \\
\text { (bilateral) }\end{array}$} \\
\hline Chi-cuadrado de Pearson & $1,480^{\mathrm{a}}$ & 3 &, 687 \\
Razón de verosimilitudes & 1,482 & 3 &, 686 \\
Asociación lineal por lineal &, 090 & 1 &, 764 \\
N de casos válidos & 45 & & \\
\hline
\end{tabular}

FUENTE: Reporte del SPSS 20,0

$$
p=0,687
$$

El valor del chi-cuadrado calculado fue de 1,480 (valor de $p=0,687$ ), de donde el valor de $p$ es superior al nivel de significancia del 5\%; ello implica que se rechaza $\mathrm{H}_{0}$; es decir, no existe una influencia significativa de la satisfacción docente sobre el desempeño laboral en el Centro de Idiomas de la UNJBG de Tacna, en el año 2014.

\section{CONCLUSIONES}

a) El trabajo de investigación se basó en la población de docentes del Centro de Idiomas de la UNJBG de Tacna, es decir, 45 personas. Se concluye que no existe una influencia significativa de la satisfacción docente sobre el desempeño laboral.

b) Se obtuvo un valor medio de 70,56; lo cual permite concluir que el personal docente del CEID - UNJBG de Tacna considera que se caracteriza por un nivel aceptable de satisfacción por la docencia que imparte en dicha entidad. Al analizar el valor medio según género, el masculino obtuvo una media de 67 y el femenino 75, es decir nivel de satisfacción regular y alto respectivamente. Asimismo, se observó que a mayor años de experiencia, menor satisfacción docente. Por indicador, se encontró que el aspecto más resaltado fue la "Supervisión", siendo los aspectos a mejorar las "Recompensas justas" y las "Condiciones de trabajo".

c) Se obtuvo un valor medio de 63,84 ; lo cual permite concluir que el personal docente del CEID - UNJBG de Tacna considera que su nivel de desempeño en sus laborales académicos, son destacadas. Al analizar el valor medio según género, el masculino obtuvo una media de 63 y el femenino 65, es decir nivel de desempeño laboral muy adecuado en ambos casos. Considerando años de experiencia, los indicadores "Crecimiento personal" y "Labor de equipo" disminuyeron con el transcurrir de los años, los dos restantes presentaron comportamiento irregular. Por indicador, se encontró que el aspecto más resaltado fue la "Satisfacción al estudiante", siendo los aspectos a mejorar la "Labor de equipo" y el "Crecimiento personal". 
d) Se concluye que no existe una relación directa y significativa entre los indicadores del nivel de satisfacción docente y los indicadores del desempeño laboral en el Centro de Idiomas de la UNJBG de Tacna.

\section{REFERENCIAS BIBLIOGRÁFICAS}

- ACTIO REPORTE. (2006). Evaluación de Desempeño. News Letter Diario, 1,2.

- AGUDELO, S. (1998). Certificación de competencias laborales. Aplicación en Gastronomía. Montevideo: Cinterfor-OIT.

- ALARCON, N., \& MENDEZ, R. (15 de Diciembre de 2012). Calidad de la Docencia. Obtenido de http://www.monografias.com/trabajos10/ponenc/ponenc.shtml

- AlvareZ BeCerRA, R. M. (15 de Abril de 2015). Metodologia de la Investigación Científica. (A. L. Dávalos, Entrevistador)

- AlvareZ-Garcia, D., ROdRigueZ, C., GONZALES-CAStro, P., NUÑEZ, J., \& ALVAREZ, L. (2010). La formación inicial de los futuros maestros en recursos para la convivencia escolar y el manejo del aula. European Journal of Education and Psychology, 187, 198.

- ARCILA, G., LOPEZ, J., \& CASTAÑO, L. (2014). Propuesta pedagógica y gerencial para mejorar el clima organizacional. Manizales, Caldas, Colombia.

- Baguer alcala, A. (2 de Diciembre de 2006). Temas de Recursos Humanos. Obtenido de http://www.trabajofreelance.com/noticia-169

- BAGUer, A. (15 de Mayo de 2014). Clima Laboral. Obtenido de $\mathrm{http} / \mathrm{www}$.gestiopolis.com/dirgp/rec/clima.htm

- BLANCO GUILLERMETY, R., \& BLANCO PECK, R. $\left(1^{\circ}\right.$ de Diciembre de 2007). La Medición de la Calidad de Servicios en la Educacion Universitaria. (E. Eduardo Aponte Hernández, Ed.) Cuaderno de Investigación en la Educación, 1(22), 121-136.

- BLUM, M., \& NAYLOR, J. (1999). Fundamentos Teoricos y Sociales de Psicologia Industrial. Barcelona.

- BLUM, M., \& NAYLOR, S. (1981). Psicología Industrial. México: Trillas.

- BUNK, G. (1994). La transmisión de las competencias en la formación y perfeccionamiento profesionales en la RFA. Revista CEDEFOP №1, 112.

- BYARS, L., \& RUE, W. (1996). Gestión de Recursos Humanos. España: Editorial Mosby, Ediciones División IRWIN- Pág. 583. 
- CERTO, S., \& PETER, J. (1997). Dirección estratégica.Madrid: Mc. GrawHill.

- CHIAvenato, I. (2001). Administración de Recursos Humanos. En Evaluación del Desempeño (págs. Pág. 297 - 339). Santa Fe de Bogotá: Editorial Mc Graw - Hill .

- DRUCKER, P. (1994). Gerencia para el Futuro. En P. Drucker, Gerencia para el Futuro. España: Grupo Editoriial Norma.

Recibido: $2 / 6 / 2015$

Aceptado para publicación:

$3 / 7 / 2015$ 